\title{
AVALIAÇÃO DE EVENTOS ANTECEDENTES E SUBSEQUENTES A COMPORTAMENTOS ADEQUADOS E INADEQUADOS NA INTERAÇÃO PROFESSOR-ALUNO
}

\author{
EVALUATION ANTECEDENT AND SUBSEQUENT EVENTS TO ADEQUATE AND INADEQUATE \\ BEHAVIORS IN THE TEACHER-STUDENT INTERACTION
}

\author{
SÔNIA MARIA MELLO NEVES ${ }^{1}$ - ORCID 0000-0001-8527-4622 \\ FERNANDA CERQUEIRA BOMFIM ${ }^{1}$ - ORCID 0000-0002-9065-087X \\ JOÃO CLAUDIO TODOROV² - ORCID 0000-0001-6308-1547 \\ FÁBIO HENRIQUE BAIA ${ }^{3}$ - ORCID 0000-0002-4084-4828 \\ ANNA CAROLINA GONÇALVES SOUZA ${ }^{1}$ - ORCID 0000-0001-8832-4752 \\ IVALDO FERREIRA DE MELO JUNIOR ${ }^{1}$ - ORCID 0000-0001-6133-6665 \\ WANESSA MAGALHAES CARNEIRO ${ }^{1}$ - ORCID 0000-0002-1388-578X \\ LAÍS MELO GIGLIO ${ }^{1}$ - ORCID 0000-0002-7886-3856

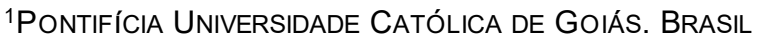 \\ 2UNIVERSIDADE DE BRASÍLIA. BRASIL \\ 3UNIVERSIDADE DE RIO VERDE E UNIVERSIDADE DE SÃo PAULO. BRASIL
}

\begin{abstract}
RESUMO
A interação professor-aluno é um aspecto determinante para aprendizagem em sala de aula. O objetivo deste estudo foi realizar a avaliação funcional da interação professor-aluno em sala de aula, em uma escola municipal com alto índice de violência. Participaram deste estudo seis estudantes com idades entre 9 a 16 anos, do $6^{\circ}$ ao $8^{\circ}$ ano do ensino fundamental, que compuseram dois grupos: Disciplinados e Indisciplinados; além de 10 professores, sendo nove mulheres e um homem. As interações entre os participantes foram registradas em vídeo. Pesquisadores analisaram os vídeos categorizando os comportamentos dos alunos como adequados e inadequados, registrando eventos anteriores e subsequentes desses comportamentos a partir de categorias de respostas dos professores. Os resultados indicam que não houve diferença de emissão de comportamentos apropriados entre alunos do Grupo Disciplinados ou do Grupo Indisciplinados. Já os comportamentos inadequados são mais frequentes entre os alunos do Grupo Indisciplinados. Foram observadas poucas situações nas quais o professor apresentava eventos subsequentes aos comportamentos dos alunos. Quando havia eventos subsequentes esses eram mais comuns para o comportamento adequado dos membros do Grupo Disciplinado e inadequados para o comportamento dos membros do Grupo Indisciplinado. Discute-se possíveis relações entre os eventos antecedentes, eventos subsequentes e comportamentos adequados e inadequados. Sugere-se treinamento aos professores para identificar relações funcionais e intervirem no comportamento de seus alunos. Além de condução de outras pesquisas que investiguem as condições de trabalho e eventos ambientais que determinam ou limitam a capacidade dos professores de intervirem no comportamento de seus alunos.
\end{abstract}

Palavras-chave: avaliação funcional; interação professor-aluno; comportamento adequado; comportamento inadequado.

\section{ABSTRACT}

The student-teacher interaction is a determinant aspect of learning in a classroom. The objective of this study was to conduct a functional evaluation of student-teacher interactions, in the classroom, in a municipal school with high levels of violence. Six students, aged between 9 and 16 years, participated in the study. They were 6th and 8th grade middle school students and they were assigned to two groups: Disciplined and Undisciplined. Ten teachers also participated, 9 of which were women and one man. The interactions between participants were recorded on camera. Researchers analyzed the videos by categorizing behaviors from students as adequate or inadequate, and recording the antecedents and subsequent events of each behavior. Results indicated no differences in emission of appropriate behaviors between students from the Disciplined and the Undisciplined groups. Few situations were observed in which the teacher presented events subsequent to the students' behavior. When there were subsequent events these were more frequent for the adequate behavior of the members of the Disciplined Group and inadequate for the behavior of the members of the Undisciplined Group. Possible relationships between antecedent events, subsequent events and appropriate and inappropriate behaviors are discussed. It is suggested that teachers need to be trained in order to be able to identify functional relationships and intervene in the behavior of their students, thus favoring the emission of appropriate behaviors. We also suggest to conducting other research that investigates working conditions and environmental events that determine or limit teachers' ability to intervene in their students' behavior.

Keywords: evaluating analysis; student-teacher interaction; adequate behavior; inadequate behavior.

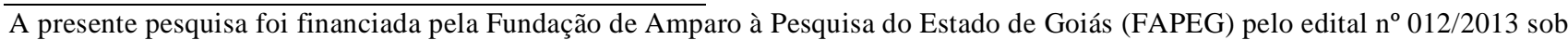
o processo 201410267000331 tendo a primeira autora como beneficiária e coordenadora geral da pesquisa. Correspondência para Fábio Henrique Baia. E-mail: fabio@unirv.edu.br DOI: http://dx.doi.org/10.18542/rebac.v16i1.8883 
O relacionamento professor-aluno exerce influência importante no ajuste acadêmico, socialemocional e comportamental de crianças (Biglan \& Glenn, 2013; Ladd, 2005). A presença de professores atenciosos e exigentes pode melhorar o senso de adesão dos estudantes e reduzir o conflito entre pares na sala de aula (Barr \& Parrett, 2001). As relações positivas professor-aluno também foram consideradas importantes para o ajuste de crianças em situação de risco (Baker, Grant, \& Morlock, 2008). Por meio da interação com professores, as crianças podem aprender lições de vida importantes sobre empatia, respeito pelos outros e resolução pacífica de conflitos (Hanish et al., 2004). A psicologia pode contribuir para o desenvolvimento de melhores condições de aprendizagem por meio de ferramentas que permitam compreender, promover e alterar comportamentos. A avaliação funcional do comportamento é uma dessas ferramentas para melhorar as condições de ensino e aprendizagem.

As avaliações funcionais do comportamento ${ }^{1}$ são comumente realizadas em contextos escolares e tipicamente incluem medidas indiretas como entrevistas e escalas, além de observações diretas (Anderson, Rodriguez, \& Campbell, 2015; Borgmeier, Loman, Hara, \& Rodriguez, 2015). Ao longo dos últimos anos, um crescente corpo de pesquisas se concentrou no uso de avaliação funcional em ambientes escolares (Gage, Lewis, \& Stichter 2012; Solnick \& Ardoin, 2010). Parte desse crescimento é sustentado pela sugestão de que estratégias de intervenção baseadas em avaliações funcionais produzem resultados superiores aos efeitos produzidos por intervenções escolares típicas, isto é, aquelas que não utilizam análise funcional do comportamento (McIntosh, Brown, \& Borgmeier, 2008).

Scott, Alter e Hirn (2011) investigaram se os comportamentos dos professores afetavam a taxa de sucesso dos alunos. Os alunos foram categorizados em dois grupos: (i) Estudantes que haviam sido previamente diagnosticados com Transtornos de Comportamentos Disruptivos (TDR) e (ii) Estudantes sem Transtornos. Foram realizadas mais de 1000 observações em sala de aula. Nessas observações foram registrados os comportamentos dos professores, os comportamentos dos alunos e as consequências liberadas. Os resultados encontrados indicam que os professores tendiam a oferecer maior quantidade de oportunidade de participação para o grupo TDR - sem especificar qual aluno deveria responder - do que individualmente. Ademais, houve menos oportunidades (professor requisitar individualmente à um aluno responder uma questão ou apresentar um conteúdo acadêmico) de participação para alunos membros do grupo com TDR quando comparado às oportunidades oferecidas aos

\footnotetext{
${ }^{1} \mathrm{O}$ termo avaliação é aqui empregado no sentido proposto por Sturmey (1996). O autor sugere o uso do termo para situações nas quais se avalie - de modo indireto - possíveis relações funcionais de comportamentos. Nesse sentido a avaliação pode ser parte de uma Análise Funcional - já que esta exige a manipulação de variáveis. Assim, por avaliação funcional nos referimos a
}

estudantes sem TDR. Em relação aos tipos de consequências liberadas pelos professores, foi observado que a quantidade de feedbacks positivos apresentados pelos professores não diferiu entre os grupos. Já os feedbacks negativos foram mais frequentes para alunos que apresentavam comportamentos problema. Também foi observado que quando os alunos de ambos os grupos erguiam as mãos solicitando atenção do professor, apenas em $57 \%$ das ocorrências o professor atendeu ao chamado dos alunos (sem diferenciação entre os grupos).

Viecili e Medeiros (2002) investigaram se o fracasso escolar poderia estar correlacionado à existência de um ambiente coercitivo na escola. Participaram do estudo dez crianças (de diferentes turmas de uma escola) com idade entre 8 e 10 anos e três professoras. As crianças foram classificadas em dois grupos; com história de fracasso escolar (CFE) e sem história de fracasso escolar (SFE). O fracasso escolar foi definido como histórico de repetência. O procedimento para análise de dados envolveu registro cursivo para desenvolvimento de categorias; categorização dos comportamentos; novas sessões de observação com registro de ocorrências dos comportamentos por categorias.

Os resultados encontrados por Viecili e Medeiros (2002) indicam que houve maior ocorrência de estimulação aversiva por parte dos professores para comportamentos emitidos por alunos do grupo CEF. Ademais, os professores tendiam a liberar consequências, como elogios, mais frequentemente para alunos do grupo SFE. Em relação ao comportamento dos alunos, foi observado que os membros do grupo SFE tendiam a se engajar de modo mais atento nas tarefas propostas pela professora. Já os alunos do grupo CFE tendiam a emitir comportamentos não relacionados às atividades propostas em sala de aula. As autoras concluem que os professores tendiam a ser mais coercitivos com os alunos do grupo CFE, e que o uso de punição pode ter sido uma variável que aumentava a emissão de comportamentos não relacionados a atividades escolares. Nessa interpretação os comportamentos não relacionados a atividades escolares ocorreriam como contra-controle ao controle aversivo utilizado pela professora.

Outra pesquisa interessada na relação do comportamento de professores e alunos foi realizada por Guimarães e Luna (2016). O objetivo do estudo foi verificar se uma intervenção - caracterizada por apresentação sistemática de vídeos da interação professora e aluna, feedbacks sobre variáveis explicativas dos comportamentos e sugestões de comportamentos alternativos - produziria modificação do comportamento da professora. As aulas da professora

interpretações de possíveis relações entre eventos ambientais e respostas extraídas dos registros em vídeo de interações em sala de aula sem que tenha ocorrido à manipulação de variáveis que comprovaria a relação funcional. 
foram registradas em vídeo. Os comportamentos da aluna foram categorizados como Participar - respostas relacionadas às atividades propostas pela professora - e Outros comportamentos. Já os comportamentos da professora foram categorizados como Perguntar, Explicar, Consequenciar, entre outras. Imagens dos registros em vídeos foram apresentados para professora em conjunto com questionamentos, feedbacks e orientações. Em seguida foram registradas novas aulas da professora e novas rodadas de intervenção.

Os resultados indicaram que inicialmente as categorias de comportamento da professora mais frequentes foram Perguntar e Explicar. Já as menos frequentes envolviam Consequenciar e Criar Condições (para o comportamento da aluna). Além disso, foi observado que nas últimas aulas registradas a professora emitiu, com maior frequência, comportamentos relacionados ao comportamento da aluna, incluindo dar feedbacks e dicas sobre como desempenhar a tarefa proposta. Esses resultados sugerem que o comportamento da professora pode ser alterado utilizando os recursos de intervenção empregados pelos pesquisadores.

Considerando a relevância social do desenvolvimento de estudos utilizando a avaliação funcional como metodologia de análise de comportamentos no contexto escolar, o presente trabalho teve como objetivo realizar avaliação funcional da interação professor-aluno em sala de aula, em uma escola municipal com alto índice de violência. Foram identificadas diferentes categorias e frequências de comportamento. Também foi avaliado se havia diferenças entre eventos ambientais antecedentes e subsequentes em relação ao tipo de comportamento (se apropriado ou inapropriado à situação de aprendizagem escolar) emitido por alunos que apresentavam problemas de indisciplina e aqueles que não apresentavam esse tipo de problema.

\section{Participantes}

Participaram do estudo seis estudantes com idade entre 9 e 16 anos, matriculados em três turmas diferentes do turno matutino $\left(6^{\circ}, 7^{\circ}\right.$ e $8^{\circ}$ ano), e 10 professores - nove mulheres e um homem. Os professores participantes eram aqueles que ministravam as disciplinas nas turmas nas quais esta pesquisa foi realizada. Todos os professores de todas as disciplinas aceitaram participar desta pesquisa. A Diretora da Escola indicou três turmas com alto índice de problemas disciplinares para realização da pesquisa. Após observação sistemática, foram selecionados dois estudantes de cada turma. O critério de escolha envolveu o total de ocorrências dos comportamentos apropriados ou inapropriados.

A participação dos estudantes foi consentida por seus responsáveis legais por meio do Termo de Assentimento Livre Esclarecido (TALE) e do Termo de Consentimento Livre Esclarecido (TCLE). Todos professores assinaram o TCLE. Este estudo foi aprovado pelo Comitê de Ética da Pontifícia Universidade Católica de Goiás (CEP-PUCGO) sob o protocolo $\mathrm{n}^{\mathrm{o}}$ 34169814.5.0000.0037.

\section{Ambiente e Materiais}

A pesquisa foi realizada em escola pública com alto índice de violência escolar da cidade de Goiânia-GO. A escolha da escola foi por indicação da Secretária Municipal de Educação de Goiânia-GO. Todas as sessões ocorreram nas salas de aula (nas quais os alunos participantes estudavam) ou na sala de informática. Todas as sessões foram registradas em vídeo. Foram utilizados tablets para filmagem, canetas, lápis, computadores, folhas de registro, disco rígido externo e cartões de memória para armazenamento dos vídeos.

\section{Procedimento categorias. \\ Registro de observação sistemática e criação de}

Foram realizadas três sessões de observações nas quais foram registradas, em vídeo, as interações ocorridas em sala de aula. Cada sessão teve a duração de $20 \mathrm{~min}$. Antes das filmagens os alunos e professores foram informados dos objetivos da pesquisa. Porém, não foi informado quais alunos seriam alvo das observações. $\mathrm{O}$ objetivo desses registros foi permitir (1) a identificação de alunos para composição dos Grupo Disciplinados e Grupo Indisciplinados e (2) construção de categorias. As categorias foram construídas de modo a permitir identificar (i) eventos ambientais antecedentes; (ii) respostas e (iii) eventos ambientais subsequentes.

Os registros em vídeos foram assistidos de modo independente por dois pesquisadores que incialmente definiram três categorias comportamentais referentes ao desempenho dos alunos, a saber: comportamentos adequados, comportamentos inadequados e outros. Foi definido como comportamento adequado as respostas dos participantes que potencialmente aumentassem a probabilidade de aprendizagem. Comportamentos adequados foram operacionalizados nas subcategorias: (a) Realizar as Tarefas Acadêmicas (RTA) - por exemplo, copiar conteúdo, executar exercícios propostos pela professora, ler material solicitado, e (b) Conversar sobre a Tarefa Escolar (CTA) - caracterizado por conversar com colegas de classe sobre a temática referente à atividade proposta pela professora. Os comportamentos inadequados envolviam comportamentos que potencialmente diminuíssem a probabilidade de aprendizagem, e foram definidos nas subcategorias: (d) Indisciplina (IND) (i.e., descumprir regras escolares, rir ou conversar com o colega enquanto a professora realiza exposição de conteúdo; falar com tom irônico); (e) Violência Verbal (VV) - dizer palavras de baixo calão, ofender oralmente ou gestualmente colegas ou professores; (f) Agressão Física (AF) - chutar, beliscar, tocar outra pessoa com força desproporcional ou atirar objetos em outras pessoas e (g) Desviar atenção durante tarefa (DAT) - conversar sobre assuntos não relacionados a tarefa, olhar para outros lugares, desenhar, bocejar, dormir durante período dedicado para realização de tarefas acadêmicas. A categoria Outros foi composta por comportamentos que não se enquadravam nas categorias anteriores. Exemplos de comportamentos que compuseram a categoria Outros são: manipular materiais, redigir bilhetes, bocejar, espreguiçar durante momentos em que 
não havia demanda escolar como tarefas ou explicação da professora.

Os comportamentos dos professores foram considerados como eventos ambientais e foram operacionalizados como: (i) Explicar Conteúdo (EXP) explicar conceitos, descrever as tarefas acadêmicas a serem realizadas, escrever no quadro negro; (ii) Advertência ao Aluno (ADA) - informar ao aluno anunciando seu nome individualmente sobre comportamentos inapropriados, solicitar silêncio, anunciar castigos, solicitar que o aluno se sente ou interrompa algum comportamento; (iii) Advertência à Turma (ADT) - mesmos comportamentos descritos na advertência ao aluno, porém sem especificar a quem a advertência se dirige; (iv) Questionar (QST) - solicitar respostas relacionadas ao conteúdo acadêmico, pedir informações de quaisquer natureza, solicitar material escolar para alunos; (v) Realizar Atividades Gerais (RAG) - fazer chamada, entregar provas ou atividades, dar visto em cadernos, separar a turma em grupos; e (vi) Responder Aluno (RSP) - elucidar questionamentos individuais dos alunos.

As categorias de eventos ambientais antecedentes foram definidas como comportamentos emitidos pelos professores imediatamente antes da emissão dos comportamentos dos alunos. Já as categorias de eventos ambientais subsequentes foram compostas por comportamentos dos professores que ocorreram imediatamente após a emissão dos comportamentos dos alunos.

Após a criação das categorias comportamentais, dois pesquisadores independentes assistiram as filmagens e registraram a frequência de comportamentos emitidos por cada estudante. Para identificação do episódio comportamental, utilizou-se como indicador o momento que um estudante começava a emitir uma resposta. Após a identificação do início da resposta regressava-se a filmagem em 5 s. Foi considerado evento ambiental antecedente o comportamento da professora que antecedeu a resposta do aluno em até $5 \mathrm{~s}$. Caso não houvesse comportamentos da professora considerava-se que para aquele episódio não havia eventos antecedentes. Foram considerados eventos subsequentes os comportamentos da professora que ocorrem após o fim da resposta do aluno com latência máxima de $5 \mathrm{~s}$. Assim, um episódio comportamental poderia envolver: (a) apenas eventos antecedentes; (b) apenas eventos subsequentes; (c) envolver apenas respostas, portanto, não envolver eventos antecedentes ou subsequentes ou (d) envolver eventos antecedentes e eventos subsequentes - chamado de episódio comportamental completo.

Cada turma possuía cerca de 30 estudantes. Nessa etapa os vídeos foram filmados em um enquadramento que permitia observar todos os alunos. Foram feitos registros para cada um dos alunos da turma. Isso é, uma pesquisadora assistia o mesmo vídeo repetidas vezes, sendo que à cada observação procedia-se ao registro do comportamento de um dos alunos. O estudante de cada turma que apresentou maior frequência de comportamentos adequados foi incluído no Grupo Alunos
Disciplinados. Já o estudante de cada turma com maior frequência de comportamentos inadequados foi incluído como membro do Grupo Alunos Indisciplinados. Portanto, em cada turma havia dois estudantes participantes da pesquisa, sendo um de cada grupo. Maria, Ana e Jorge, nomes fictícios, pertenciam ao Grupo de Alunos Disciplinados e Bruno, Felipe e Vera ao Grupo de Alunos Indisciplinados.

\section{Registro Categorizado.}

Foram realizadas um total de 60 sessões de registro categorizado - 10 sessões para cada um dos seis participantes. Como havia dois alunos por turma, a pesquisadora focava individualmente em um aluno por 10 min. Depois era realizado um intervalo de 30 segundos até que nova filmagem fosse iniciada com foco no outro participante. Assim o registro dos estudantes de uma mesma turma ocorria no mesmo dia e na mesma disciplina, entretanto com intervalo de $10 \mathrm{~min}$ de diferença, o que poderia envolver diferentes atividades. A cada dia o início da filmagem era alternado entre os participantes da mesma sala. As observações ocorreram durante as mesmas disciplinas (portanto, os mesmo(as) professores ou professoras) ao longo de 30 dias letivos consecutivos. Após as filmagens serem encerradas foi realizada a categorização dos eventos por dois observadores independentes.

\section{Treino de observadores.}

O treino de observadores foi conduzido separadamente para cada uma das duas juízas. Primeiro foram apresentadas as categorias e suas definições operacionais. A pesquisadora explicou que, a cada comportamento, o observador deveria registrar na folha de registro identificando: (i) o tempo de vídeo no qual o comportamento ocorria, (ii) a qual categoria o comportamento pertencia, além dos eventos antecedentes e subsequentes envolvidos. Após a instrução foi solicitado que cada observadora realizasse a categorização de três sessões. Após a categorização das três primeiras sessões a pesquisadora se encontrava novamente com cada uma das observadoras e esclarecia eventuais dúvidas. Ao final do encontro, caso não houvesse mais dúvidas, a pesquisadora entregava os cartões de memória com os demais 57 vídeos para que os registros das demais sessões fossem categorizados.

Cálculo de Concordância entre Observadores.

O índice de acordo entre observadores $(80.96 \%)$ foi obtido pela equação:

\section{$\frac{\text { Concordâncias }}{\text { Concordâncias }+ \text { Discordâncias }} \times 100$}

Sendo que havia 902 concordâncias e 112 desacordos com um total de 1.114 eventos comportamentais (i.e., estímulos antecedentes, respostas dos alunos e eventos subsequentes). 


\section{RESULTADOS}

Foram registrados 1.114 eventos comportamentais. A Figura 1 apresenta a frequência dos comportamentos adequados, inadequados e outros de cada participante dos dois grupos. De modo geral, ambos os grupos tenderam a apresentar maior frequência de comportamentos adequados do que inadequados. Porém, a inspeção visual de Figura 1 sugere que há diferenças na frequência destes comportamentos entre grupos. Os participantes do Grupo Indisciplinados apresentaram menor diferença entre a frequência de comportamentos adequados e inadequados do que os participantes do Grupo Disciplinados. Os comportamentos da categoria Outros tiveram menor frequência do que as demais categorias.

A Figura 1 permite ainda uma análise individual sobre os tipos de comportamentos emitidos por cada participante. Maria emitiu 195 comportamentos, sendo que $97(50 \%)$ destes eram comportamentos adequados, 78 (40\%) inadequados e 20 (10\%) outros. Ana, por sua vez, emitiu 179 comportamentos, 104 (58\%) adequados, 45 (25\%) inadequados e $30(17 \%)$ outros. Jorge foi o participante do Grupo Disciplinados que apresentou maior frequência de comportamentos, totalizando 238. Destes, $138(58 \%)$ foram adequados, 79 (33\%) inadequados e 21 (9\%) outros. Assim, do Grupo Disciplinados, Maria foi a única participante a emitir comportamentos adequados abaixo de $(50 \%)$. Maria foi também a participante do grupo a apresentar maior percentual de comportamentos inadequados.

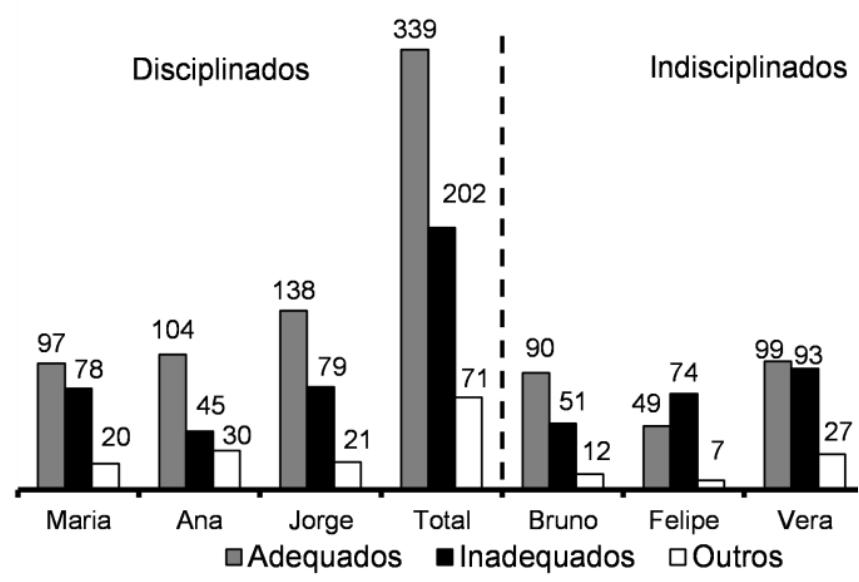

Figura 1. Frequência de emissão de comportamentos adequados e inadequado de cada aluno participante de cada grupo (disciplinado e indisciplinado).

Os participantes do Grupo Indisciplinados apresentaram padrão de comportamentos adequados e inadequados diferente do Grupo Disciplinados $\left(\chi^{2}(2)=\right.$ 12,90, $p=0,002)^{2}$. Bruno emitiu 153 comportamentos, sendo que 90 (59\%) eram adequados, 51 (33\%) inadequados e $12(8 \%)$ outros. Foram registrados 130 comportamentos de Felipe, sendo 49 (38\%) adequados, 74
(57\%) inadequados e $7(5 \%)$ outros. Vera emitiu um total de 219 comportamentos, 99 (45\%) adequados, 93 (42\%) inadequados e 27 (13\%) outros. Assim, apenas Bruno emitiu comportamentos adequados acima de 50\%. Em relação aos comportamentos inadequados, Bruno foi o que apresentou menor porcentagem de comportamentos desta categoria. $\mathrm{O}$ valor proporcional de comportamentos adequados apresentado por Bruno foi o maior valor apresentado por participantes do Grupo Disciplinados.

A Figura 2 apresenta os dados referentes a comportamentos adequados dos dois grupos. No painel superior são apresentados os eventos antecedentes a tais comportamentos. No painel inferior são apresentados as categorias comportamentais e os eventos subsequentes. A porcentagem de comportamentos adequados nos quais foi possível identificar eventos ambientais antecedentes emitidos pela professora variou entre $44 \%$ e $58 \%$ para cinco participantes (Maria, Ana, Jorge, Bruno e Felipe), para Vera a porcentagem foi de $87 \%$. Para Maria, foi possível identificar $46 \%$ dos comportamentos adequados com eventos ambientais antecedentes. Destes, 39\% ocorreram após explicações da professora sobre a tarefa EXP, 3\% após a professora realizar tarefas gerais (RAG), outros $2 \%$ envolveram questionamentos da professora (QST) e, por fim, 2\% advertências para a turma (ADT). Para Ana, 31\% foram emitidos após EXP e $27 \%$ após RAG. Dos comportamentos emitidos por Jorge, $47 \%$ ocorreram após EXP e $16 \%$ após RAG. Em relação aos participantes do Grupo Indisciplinados, Bruno emitiu 20\% dos comportamentos após EXP, 13\% ocorreram depois de QST e $8 \%$ sucederam RAG. Felipe emitiu $20 \%$ de seus comportamentos após EXP e 14\% após RAG. Vera apresentou $76 \%$ dos comportamentos após a professora realizar EXP.

O painel inferior da Figura 2 apresenta os eventos subsequentes. Observa-se que os comportamentos adequados foram significativamente mais frequentes no Grupo Disciplinados $\left(\chi^{2}(1)=4,77, p=0,029\right)$. No painel inferior, à esquerda, é possível observar que dos 97 comportamentos adequados emitidos por Maria, em apenas $9(9 \%)$ foi possível identificar algum evento subsequente que envolvia o comportamento da professora. Destes, 6\% tiveram como eventos subsequentes a explicação da professora (EXP) produzidas pelos comportamentos: (a) realização de tarefas acadêmicas (RTA) e (b) Conversa sobre a tarefa acadêmica (CTA). Ana emitiu 104 comportamentos apropriados, dos quais $9(9 \%)$ foram seguidos por respostas da professora (RSP) à aluna. Tais respostas ocorreram após RTA e CTA. Jorge por sua vez apresentou um total de 138 comportamentos apropriados, dos quais 31 (23\%) tiveram eventos subsequentes (respostas da professora) identificados. Desses, 25 (19\%) eventos comportamentais envolveram as categorias RTA e CTA e foram seguidos por EXP. De modo geral foi observado que RTA com eventos subsequentes EXP foi mais frequente para dois dos três participantes do Grupo

\footnotetext{
${ }^{2}$ Para o cálculo do Qui-quadrado $\left(\chi^{2}\right)$ utilizamos medidas de porcentagens.
} 
Disciplinados. Vale destacar que considerando o total de comportamentos apropriados, eventos subsequentes ocorreram em menos de $15 \%$ das oportunidades para quatro participantes (Maria, Ana, Bruno e Vera), e em menos de $25 \%$ das oportunidades para dois participantes (Jorge e Felipe).

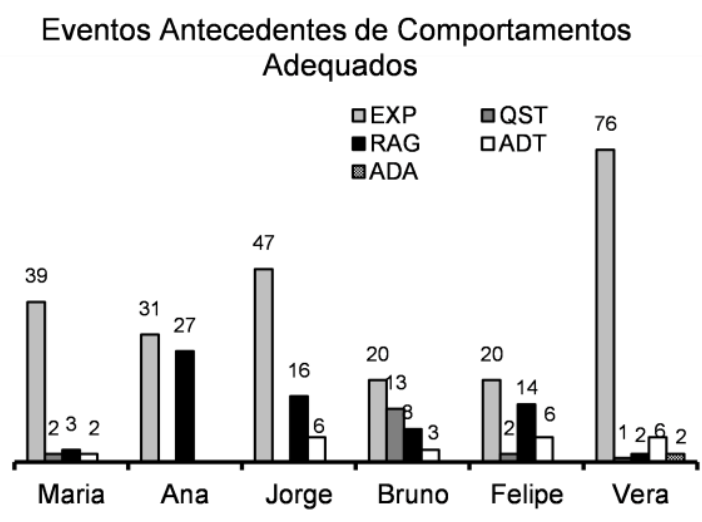

Respostas Adequadas e Eventos Subsequentes

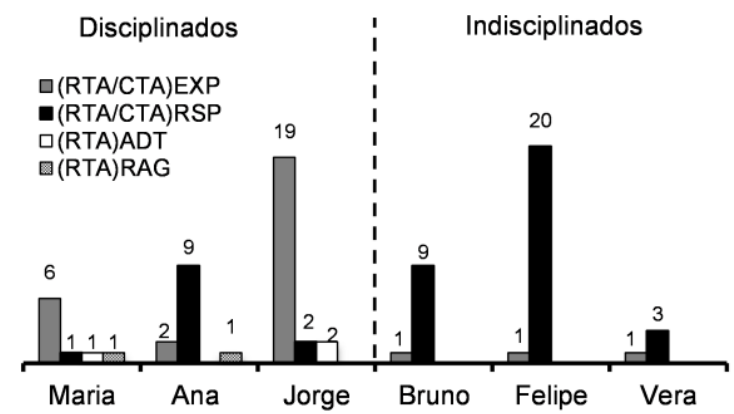

Figura 2. Porcentagem de comportamentos adequados por eventos antecedentes (painel superior) e eventos subsequentes (painel inferior) para cada aluno.

As relações (RTA/CTA)RSP foram identificadas com maiores porcentagens dentre os comportamentos adequados para os três participantes do Grupo Indisciplinados (Bruno, Felipe e Vera). Bruno emitiu 90 comportamentos adequados dos quais em 9 (10\%) foram identificados eventos subsequentes. Para esse aluno, a categoria com maior porcentagem foi (RTA/CTA) que teve como eventos subsequentes a professora responder dúvidas do aluno diretamente (RSP) com 8 ocorrências (9\%). Felipe emitiu 49 comportamentos adequados. $\mathrm{Na}$ análise dos vídeos foi possível identificar 12 (21\%) comportamentos adequados com eventos subsequentes. Destes, em 20\% foram observadas as relações (RTA/CTA)RSP. Para os comportamentos adequados de Vera, apenas 4 dos 99 episódios registrados tiveram eventos subsequentes registrados (4\%), sendo $3(3 \%)$ da classe (RTA/CTA)RSP. Vale destacar ainda que a análise entre grupos permite observar que os alunos do Grupo Disciplinado tiveram a classe (RTA/CTA)EXP mais frequente entre as respostas adequadas. Já os alunos do Grupo Indisciplinados tiveram (RTA/CTA)RSP como o tipo mais frequente.
A Figura 3, apresenta os dados referentes aos comportamentos inadequados dos dois grupos. No painel superior são apresentados os eventos antecedentes a tais comportamentos. No painel inferior são apresentados as categorias comportamentais e os eventos subsequentes. É possível observar na figura que a porcentagem de comportamentos inadequados cujos eventos antecedentes envolviam respostas da professora foi de $27 \%$ para Bruno, $45 \%$ para Felipe e $99 \%$ para Vera. Para os membros do Grupo Disciplinados (Maria, Ana e Jorge) os valores variaram entre $85 \%$ e $100 \%$. Para todos os participantes, o evento antecedente predominantemente mais comum para comportamentos inadequados foram as explicações da professora (EXP). O painel inferior da Figura 3 apresenta a porcentagem de respostas para comportamentos inadequados dos participantes deste estudo, com apresentação significativamente maior de eventos subsequentes para o Grupo Indisciplinado $\left(\chi^{2}(1)=5,51, p=\right.$ 0,019). Todos os participantes do Grupo Disciplinado apresentaram comportamentos das categorias desviar atenção durante tarefa (DAT); indisciplina (IND); violência verbal (VV), que tiveram como eventos subsequentes a professora advertir a turma (ADT).

\section{Eventos Antecedentes de Comportamentos} Inadequados

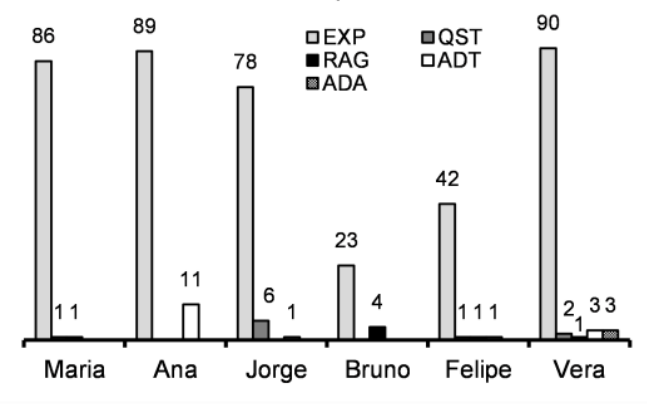

Respostas Inadequadas e Eventos Subsequentes

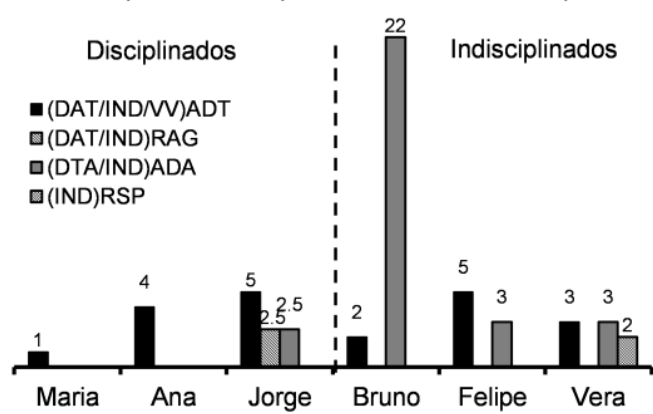

Figura 3. Porcentagem de comportamentos inadequados por eventos antecedentes (painel superior) e eventos subsequentes (painel inferior) para cada aluno.

Dos 78 comportamentos inadequados de Maria, apenas em 1 (1\%) o evento subsequente foi a professora advertir a turma (ADT). Ana emitiu 45 comportamentos inadequados, sendo que $2(4 \%)$ tiveram como evento subsequente ADT. Jorge emitiu 79 comportamentos inadequados, destes, em $8(10 \%)$ foi possível identificar eventos subsequentes. Dos 8, 4 (5\%) eram do tipo (DAT/IND/VV)ADT, 2 (2,5\%) (DAT/IND) tiveram como evento subsequente a advertência ao aluno individualmente 
(ADA) e $2(2,5 \%)$ a realização de atividades gerais (RAG). Já os participantes do Grupo Indisciplinados não apresentaram um padrão sistemático. Bruno emitiu 51 comportamentos inadequados, dos quais 12 (24\%) foram identificados eventos subsequentes liberados pela professora. Destes, 11 (22\%) do tipo (DAT/IND)ADA e 1 (2\%) (DAT/IND/VV)ADT. Felipe emitiu 74 comportamentos inadequados, sendo $6(8 \%)$ tendo eventos subsequentes apresentados pela professora. Destes, $4(5 \%)$ do tipo (DAT/IND/VV)ADT e 2 (3\%) (DAT/IND)ADA. Vera se engajou em um total de 93 episódios classificados como inadequados, dos quais $8(8 \%)$ tiveram eventos subsequentes identificados. Sendo $3(3 \%)$ do tipo (DAT/IND/VV)ADT, 3 (3\%) (DAT/IND)ADA e 2 (2\%) da classe indisciplina (IND), que produziram como evento subsequente a professora emitir respostas a dúvidas diretamente ao aluno (RSP).

A análise completa de eventos antecedentes (contexto), comportamentos e eventos subsequentes para comportamentos apropriados e inapropriados é apresentada na Tabela 1. Para a análise, são apresentados os episódios em que foi possível identificar tanto o contexto como os eventos subsequentes do comportamento. Dos 1.114 episódios analisados, apenas em $54(4,8 \%)$ foi possível realizar a análise completa dos eventos antecedentes e subsequentes dos comportamentos, já que nos demais 1.160 foi possível observar apenas eventos antecedentes ou subsequentes. Desses 54 eventos nos quais foi possível observar eventos antecedentes, comportamentos dos alunos e eventos subsequentes, 42 $(77,8 \%)$ foram comportamentos adequados e 12 (22,2\%) inadequados. Os participantes do Grupo Disciplinado emitiram 35 dos comportamentos apropriados cuja análise completa foi possível (Maria 4, Ana 4 e Jorge 27). Os participantes do Grupo Indisciplinados tiveram 7 comportamentos cuja análise foi completa (Bruno 2, Felipe 1 e Vera 4). Em geral, os comportamentos apropriados cuja análise completa foi realizada, envolveram o comportamento de realizar tarefas acadêmicas. Foram 39 episódios de comportamentos adequados envolvendo RTA. Como pode ser observado na Tabela 1, os eventos ambientais relacionados a RTA geralmente envolviam explicações (EXP) e respostas (RSP) da professora. Em relação aos comportamentos inadequados, dos 12 episódios analisados, 6 (50\%) foram emitidos por participantes do Grupo Disciplinados, e 6 (50\%) emitidos por participantes do Grupo Indisciplinados. Em geral, os comportamentos do tipo indisciplinados (IND) foram antecedidos por EXP e QST da professora para ambos os grupos. Membros do Grupo Disciplinados apresentaram 3 comportamentos do tipo distração durante a tarefa (DAT) e 3 do tipo indisciplina (IND). Já no Grupo Indisciplinados, todos os comportamentos inadequados dos quais foram possíveis análises completas envolveram indisciplina (IND).

Tabela 1.

Descrição Completa (Contexto, Resposta, Consequência) de Episódios Comportamentais e sua Frequência por Aluno dos Grupos Disciplinados e Indisciplinados.

\begin{tabular}{|c|c|c|c|c|c|c|c|c|}
\hline \multirow[b]{2}{*}{ Aluno } & \multicolumn{4}{|c|}{ Adequados } & \multicolumn{4}{|c|}{ Inadequados } \\
\hline & Antec. & Cpto. & Conseq. & Freq. & Antec. & Cpto. & Conseq. & Freq. \\
\hline \multicolumn{9}{|c|}{ Grupo de Alunos Disciplinados } \\
\hline \multirow[t]{4}{*}{ Maria } & RAG & RTA & EXP & 1 & EXP & DAT & ADT & 1 \\
\hline & QST & RTA & EXP & 1 & & & & \\
\hline & EXP & RTA & ATP & 1 & & & & \\
\hline & ADT & RTA & EXP & 1 & & & & \\
\hline \multirow[t]{4}{*}{ Ana } & RAG & CTA & EXP & 1 & EXP & IND & ADT & 2 \\
\hline & RAG & CTA & RSP & 1 & & & & \\
\hline & RAG & RTA & RSP & 1 & & & & \\
\hline & EXP & RTA & RSP & 1 & & & & \\
\hline \multirow[t]{4}{*}{ Jorge } & EXP & RTA & EXP & 12 & QST & DAT & ADT & 1 \\
\hline & QST & RTA & EXP & 12 & EXP & IND & RAG & 1 \\
\hline & RAG & RTA & ATP & 2 & QST & DAT & RAG & 1 \\
\hline & EXP & RTA & ATP & 1 & & & & \\
\hline Total & \multicolumn{7}{|c|}{35} & 6 \\
\hline \multicolumn{9}{|c|}{ Grupo de Alunos Indisciplinados } \\
\hline \multirow[t]{2}{*}{ Bruno } & RAG & CTA & RSP & 1 & & & & \\
\hline & EXP & CTA & RSP & 1 & & & & \\
\hline \multirow[t]{2}{*}{ Felipe } & EXP & RTA & EXP & 1 & EXP & IND & ADT & 2 \\
\hline & & & & & EXP & IND & ADA & 1 \\
\hline \multirow[t]{3}{*}{ Vera } & ADT & RTA & EXP & 1 & EXP & IND & RSP & 1 \\
\hline & EXP & RTA & RSP & 2 & QST & IND & $\mathrm{ADA}$ & 1 \\
\hline & ADA & RTA & RSP & 1 & EXP & IND & ADA & 1 \\
\hline Total & & & & 7 & & & & 6 \\
\hline
\end{tabular}


Nenhum dos comportamentos do tipo indisciplina do Grupo Disciplinados envolveu advertência ao aluno individualmente (ADA), no entanto em $4(66,7 \%)$ a professora advertiu a turma (ADT). Os comportamentos do tipo indisciplina do Grupo Indisciplinados tiveram como eventos subsequentes ADA em 3 (50\%) ocasiões, e ADT em $2(40 \%)$.

\section{DISCUSSÃO}

O objetivo deste estudo foi realizar uma avaliação funcional da interação professor-aluno na emissão de comportamentos adequados $\mathrm{e}$ inadequados. $\mathrm{O}$ termo avaliação funcional é aqui empregado no sentido apontado por Sturmey (1996), como um modo de avaliar indiretamente as possíveis relações entre eventos ambientais e eventos comportamentais. De modo geral os resultados indicam que os alunos (de ambos os grupos) tendiam a emitir mais comportamentos adequados do que inadequados. Entretanto, como pode ser observado na Figura 1, a diferença entre o total de comportamentos adequados e inadequados entre os grupos é perceptível. Enquanto o Grupo Disciplinado apresentou 339 comportamentos adequados e 202 inadequados - uma diferença de 137 comportamentos - o Grupo Indisciplinado totalizou 238 comportamentos adequados e 218 inadequados - uma diferença de apenas 20 comportamentos. Tal diferença, na comparação entre comportamentos adequados e inadequados, sugere que é possível haver diferentes eventos ambientais no controle dos comportamentos dos membros dos dois grupos. Essa apresentação diferencial pode explicar o porquê da razão observada entre comportamentos adequados e inadequados. Scott et al. (2011), por exemplo, observaram que as diferenças entre frequências de comportamento entre grupos - com Transtornos Disruptivos de Comportamento e sem transtornos - deviam-se a diferentes oportunidades apresentadas pelos professores.

Análises dos resultados individuais também indicam que os participantes do Grupo Disciplinados e do Grupo Indisciplinados (com exceção de Felipe) apresentaram maior frequência de comportamentos adequados do que inadequados. Em função do método utilizado - a divisão dos participantes em grupos dada a frequência de comportamentos previamente observados - a expectativa era de que membros do Grupo Disciplinados apresentassem maior porcentagem de comportamentos adequados e que membros do Grupo Indisciplinados apresentarem maior porcentagem de comportamentos inadequados. Tal expectativa decorre, além do método utilizado pelo fato, de que em estudos anteriores membros de grupos com transtornos comportamentais (e.g., Scott et al., 2011) ou classificados como emissores de comportamento problema (Wehby, Symons, \& Shores, 1995) tendiam a apresentar maior frequência de comportamentos inadequados do que os membros dos demais grupos. Não está claro por qual razão, com exceção de Felipe, os membros do Grupo Indisciplinados apresentaram maior frequência de comportamentos adequados do que inadequados. As análises dos eventos antecedentes e subsequentes não revelaram qualquer padrão que fornecesse explicação para os resultados observados, como pode ser observado a seguir.

Em relação às oportunidades oferecidas para a emissão de respostas, foi identificado maior número de classes de eventos antecedentes produzidas pelos professores para os comportamentos dos alunos disciplinados do que para os alunos indisciplinados. É possível que professores tendam a se atentar mais aos (i) comportamentos adequados dos alunos mais disciplinados e (ii) comportamentos inadequados dos alunos indisciplinados. Em Scott et al. (2011), as oportunidades para responder que o professor fornece ao grupo ou a um aluno individual foram identificadas como um comportamento positivamente correlacionado com a melhoria dos resultados acadêmicos e comportamentais dos alunos. Ainda, segundo os autores, infelizmente, as taxas de oportunidades para responder (para estudantes com problemas comportamentais e emocionais) é um fenômeno frequentemente observado na literatura que utiliza observação direta em configurações de sala de aula convencional.

Os comportamentos de explicar da professora seja um conteúdo ou uma tarefa - foi o evento antecedente com maior porcentagem tanto para comportamentos adequados quanto inadequados (ver Figuras 2 e 3). Por um lado, se espera que os comportamentos adequados dos alunos estejam sob controle do comportamento da professora. Por outro, é curioso notar que este mesmo evento esteve frequentemente e contiguamente relacionado à comportamentos inadequados. Possivelmente, as estratégias utilizadas pela professora para explicar pudessem ser aperfeiçoadas de modo a garantir maior controle do comportamento de seus alunos. Guimarães e Luna (2016) foram capazes de ampliar a capacidade de uma professora no controle do comportamento de estudantes ao implementar uma intervenção. Em estudos futuros é sugerido investigar também as condições de trabalho e outros eventos ambientais que possam ser determinantes do comportamento dos professores. A quantidade de alunos em sala, a demanda de atendimento ao conteúdo programático em tempo especificado por agências de Governo, podem ser elementos que impedem que os professores sejam capazes de controlar o comportamento dos alunos de modo efetivo para garantir a aprendizagem.

Em relação aos eventos subsequentes, foram poucas as ocasiões em que os professores emitiram os comportamentos categorizados imediatamente após os comportamentos de interesse dos(as) alunos(as). Entretanto, é possível notar na Figura 2, que em relação a comportamentos adequados, para dois dos três membros do Grupo Disciplinados, as relações (RTA/CTA)EXP foram a mais frequentes. Já para os membros do Grupo Indisciplinado a relação (RTA/CTA)RSP foi a mais frequente para todos os membros. Esses resultados sugerem possivelmente que os alunos Disciplinados possuem mais facilidade de compreender as explicações e demandas da professora. Já os alunos Indisciplinados carecem de maiores esclarecimentos e por isso, teriam como eventos 
subsequentes respostas da professora direcionadas ao aluno (RSP). Em relação aos comportamentos inadequados, observa-se que, em geral, tais comportamentos tiveram como eventos subsequentes advertências para turma (ADT) por parte da professora. Entretanto, para membros do Grupo Indisciplinados nota-se (Figura 3) que tais advertências eram direcionadas individualmente para todos os três membros do grupo, enquanto no Grupo Disciplinado apenas Jorge recebeu advertências individualizadas. É possível que os professores atentem mais aos comportamentos inadequados dos alunos indisciplinados e utilizem eventos aversivos mais direcionados, de modo a reduzir a frequências destes. $\mathrm{O}$ uso de estimulação aversiva em maior frequência para comportamentos de alunos que apresentam problemas de comportamento foi observado em outros estudos (e.g., Van Acker, Grant, \& Henry, 1996; BolsoniSilva, Mariano, Loureiro, \& Bonaccorsi, 2013).

Em apenas 4,8\% dos episódios foi possível realizar a análise completa dos eventos. A metade desses episódios foi identificada na relação entre um aluno disciplinado e professor. Essas interações foram produzidas em uma situação de realização de tarefas acadêmicas e os eventos subsequentes envolveram a resposta de "explicar" (EXP) pelo professor. Novamente o método empregado neste estudo pode ter limitado a possibilidade de registro adequado dos comportamentos. Em estudos futuros sugere-se o uso de estratégias similares às empregadas por Guimarães e Luna (2017).

Os resultados obtidos no presente estudo e suas interpretações devem ser considerados com ressalvas dadas as limitações do mesmo. Uma das principais limitações envolve a estratégia de registro por frequência dos comportamentos (e sua conversão para porcentagens). Esse tipo de registro pode ter produzido medidas observacionais menos comparáveis. O mais adequado seria registrar os comportamentos por duração e não frequência (e medidas relativas). Outra limitação é que o grau de concordância obtido foi de $80 \%$ o que implica em considerar que diferenças entre os resultados obtidos neste estudo e aqueles observados na literatura, caso ultrapassem $20 \%$, devem ser ponderados. Dito isso, é possível afirmar que os resultados aqui obtidos corroboram os achados de Wehby et al. (1995), onde as taxas de elogios dos professores em direção a alunos eram muito baixas e havia apenas uma pequena proporção de antecedentes e consequências para o estudante alvo, que emitia comportamentos problema. Wehby et al. discutiram a possibilidade de os comportamentos agressivos serem induzidos por esquemas intermitentes de reforço. Estudos apontam também que algumas consequências por exemplo, a professora parar a aula e direcionar reclamações para um aluno ou grupo de alunos - para comportamentos inadequados provavelmente aumentam a probabilidade desses no futuro, pois implicam em atenção social (Hagopian, Fisher, \& Legacy, 1994; Viecili e Medeiros, 2002). Diante dos dados obtidos na presente pesquisa, por meio do uso da análise de eventos antecedentes, comportamento dos alunos e eventos subsequentes, é sugerido o treinamento de habilidades de identificação e condução de análises funcionais por parte de professores. Tal treinamento pode ajudar a promover um ambiente escolar que favoreça comportamentos adequados. Biglan e Glenn (2013) descrevem formas de desenvolver comportamentos pró-sociais (comportamento que contribui positivamente para a sociedade e/ou o próprio indivíduo), nos diversos setores da sociedade, como a família e a escola, ressaltando a importância de reforçar os comportamentos pró-sociais e seu reconhecimento pela sociedade.

\section{DECLARAÇÃO DE CONFLITO DE INTERESSES}

Os autores declaram que não há conflito de interesses relativos à publicação deste artigo.

\section{CONTRIBUIÇÃO DE CADA AUTOR}

Certificamos que todos os autores participaram suficientemente do trabalho para tornar pública sua responsabilidade pelo conteúdo. A contribuição de cada autor pode ser atribuída como se segue: S. M. M. Neves foi a coordenadora geral da pesquisa. Em conjunto S. M. M. Neves, J. C. Todorov e F. H. Baia participaram no desenvolvimento da proposta de pesquisa, análise de dados e redação do manuscrito inicial e final. F. S Cerqueira foi a responsável por organizar e coordenar a coleta de dados, além de participar da análise de dados e redação do manuscrito inicial. A. C. G. Souza, I. F. Melo Junior, W. M. Carneiro e L. M. Giglio participaram da análise e da coleta de dados.

\section{DIREITOS AUTORAIS}

Este é um artigo aberto e pode ser reproduzido livremente, distribuído, transmitido ou modificado, por qualquer pessoa desde que usado sem fins comerciais. $\mathrm{O}$ trabalho é disponibilizado sob a licença Creative Commons 4.0 BY-NC.

\section{(cc) BY-NC}

\section{REFERÊNCIAS}

Anderson, C. M., Rodriguez, B. J., \& Campbell, A. (2015). Functional behavior assessment in schools: Current status and future directions. Journal of Behavioral Education, 24(3), 338-371. doi: 10.1007/s10864-0159226-Z

Baker, J. A., Grant, S., \& Morlock, L. (2008). The teacherstudent relationship as a developmental context for children with internalizing or externalizing behavior problems. School Psychology Quarterly, 23(1), 3-15. doi: 10.1037/1045-3830.23.1.3

Barr, R. D., \& Parrett, W. (2001). Hope fulfilled for at-risk and violent youth: $K-12$ programs that work (2a ed.). Needham Heights: Allyn and Bacon.

Biglan, A., \& Glenn, S. S. (2013). Toward prosocial behavior and environments: behavioral and cultural contingencies in a public health framework. In G. J. Madden (Ed.), APA Handbook of Behavior Analysis (pp. 255-275). Washington: American Psychological Association. 
Bolsoni-Silva, A. T., Mariano, M. L., Loureiro, S. R., \& Bonaccorsi, C. (2013). Contexto escolar: Práticas educativas do professor, comportamento e habilidades sociais infantis. Psicologia Escolar e Educacional, 17(2), 259-269. doi: 10.1590/S141385572013000200008

Borgmeier, C., Loman, S. L., Hara, M., \& Rodriguez, B. J. (2015). Training school personnel to identify interventions based on functional behavioral assessment. Journal of Emotional and Behavioral Disorders, 23,78-89. doi: 10.1177/1063426614528244

Gage, N., Lewis, T., \& Stichter, J. (2012). Behavioral assessment-based interventions with or at risk for emotional and/or behavioral disorders in school: A hierarchical linear modeling meta-analysis. Behavioral Disorders, $37,55-77$.

doi: $10.1177 / 019874291203700201$

Guimarães, L., \& Luna, S. (2016). O Comportamento do professor sob controle do comportamento do aluno: Uma intervenção em ambiente natural. Perspectivas em Análise do Comportamento, 7(2), 256-273. doi: 10.18761/pac.2016.033

Hagopian, L. P., Fisher W. W., \& Legacy S. M. (1994). Schedule effects of noncontingent reinforcement on attention-maintained destructive behavior in identical quadruplets. Journal of Applied Behavior Analysis, 27, 317-325. doi: 10.1901/jaba.1994.27-317

Hanish, L. D., Eisenberg N., Fabes, R. A., Spinrad, T. L., Ryan, P. \& Schmidt, S. (2004). The expression and regulation of negative emotions: Risk factors for young children's peer victimization. Development and Psychopathology, 16, 334-353. doi: 10.1017/S0954579404044542

Ladd, G. W. (2005). Children's peer relationships and social competence: A century of progress. New Haven: Yale University Press.

McIntosh, K., Brown, J. A., \& Borgmeier, C. J. (2008). Validity of functional behavior assessment within a response to intervention framework: Evidence, recommended practice, and future directions. Assessment for Effective Intervention, 34, 6-14. doi: $10.1177 \% 2 \mathrm{~F} 1534508408314096$

Scott, T. M., Alter, P. J., \& Hirn, R. G. (2011). An Examination of Typical Classroom Context and Instruction for Students With and Without Behavioral Disorders. Education and Treatment of Children, 34(4), 619-641. doi: 10.1353/etc.2011.0039

Solnick, M. D., \& Ardoin, S. P. (2010). A quantitative review of functional analysis procedures in public school settings. Education and Treatment of Children, 33(1), 153-175. doi: 10.1353/etc.0.0083

Sturmey, P. (1996). Functional analysis in clinical psychology. New York: Jhon Wiley \& Sons, Inc

Van Acker, R., Grant, S. H., \& Henry, D. (1996). Teacher and student behavior as a function of risk for aggression. Education and Treatment of Children, 19, 316-334.

Viecili, J., \& Medeiros, J. G. (2002). A coerção e suas implicações na relação professor-aluno. Psico-USF, 07(2), 229-238. doi: 10.1590/S141382712002000200012
Wehby, J. H., Symons, F., \& Shores, R. E. (1995). A descriptive analysis of aggressive behavior in classrooms for students with emotional and behavioral disorders. Behavioral Disorders, 20, 87-105. doi: $10.1177 / 019874299502000207$

Submetido em: 03/02/2020 Aceito em: 13/05/2020 